\title{
Approches pour une meilleure collaboration
}

\author{
Christian Bolligera, Marius Féraud ${ }^{b}$ \\ ${ }^{a}$ Dr rer. soc., chef de projet, Bureau Vatter, recherche et conseil politique, Berne \\ ${ }^{b}$ Lic. rer. soc., chef de projet, Bureau Vatter, recherche et conseil politique, Berne
}

Une bonne collaboration entre les offices AI et les médecins traitants est dans l'intérêt des patients. Elle contribue à l'efficacité de la procédure AI, à sa coordination avec le traitement médical et à l'adéquation de ses résultats. Après avoir analysé les problèmes existants et leurs causes, une étude récente a identifié un ensemble d'approches possibles pour améliorer la collaboration.

Pour l'assurance-invalidité (AI), la qualité de la collaboration avec les médecins traitants joue un rôle essentiel dans le processus de réadaptation des assurés et dans l'examen de leurs droits aux prestations. Les médecins traitants possèdent en effet des informations précieuses sur l'état de santé de leurs patients et les ressources qui peuvent être mobilisées. Eux aussi ont intérêt à une bonne collaboration avec l'AI: ils souhaitent à la fois obtenir des informations sur le déroulement de la procédure et parvenir à une bonne coordination de la procédure AI avec le traitement médical.

Sur mandat de l'Office fédéral des assurances sociales, le bureau Vatter a étudié la collaboration entre les offices AI et les médecins traitants [1]. L'étude repose sur une enquête écrite réalisée auprès des 26 offices AI cantonaux et de 325 médecins, sur une analyse comparative de l'organisation, des instruments et des processus de cinq offices $\mathrm{AI}$, ainsi que sur des entretiens en
Lire également à ce sujet les commentaires de l'Office fédéral des assurances sociales et d'un médecin spécialiste en psychiatrie et psychothérapie aux pages suivantes.

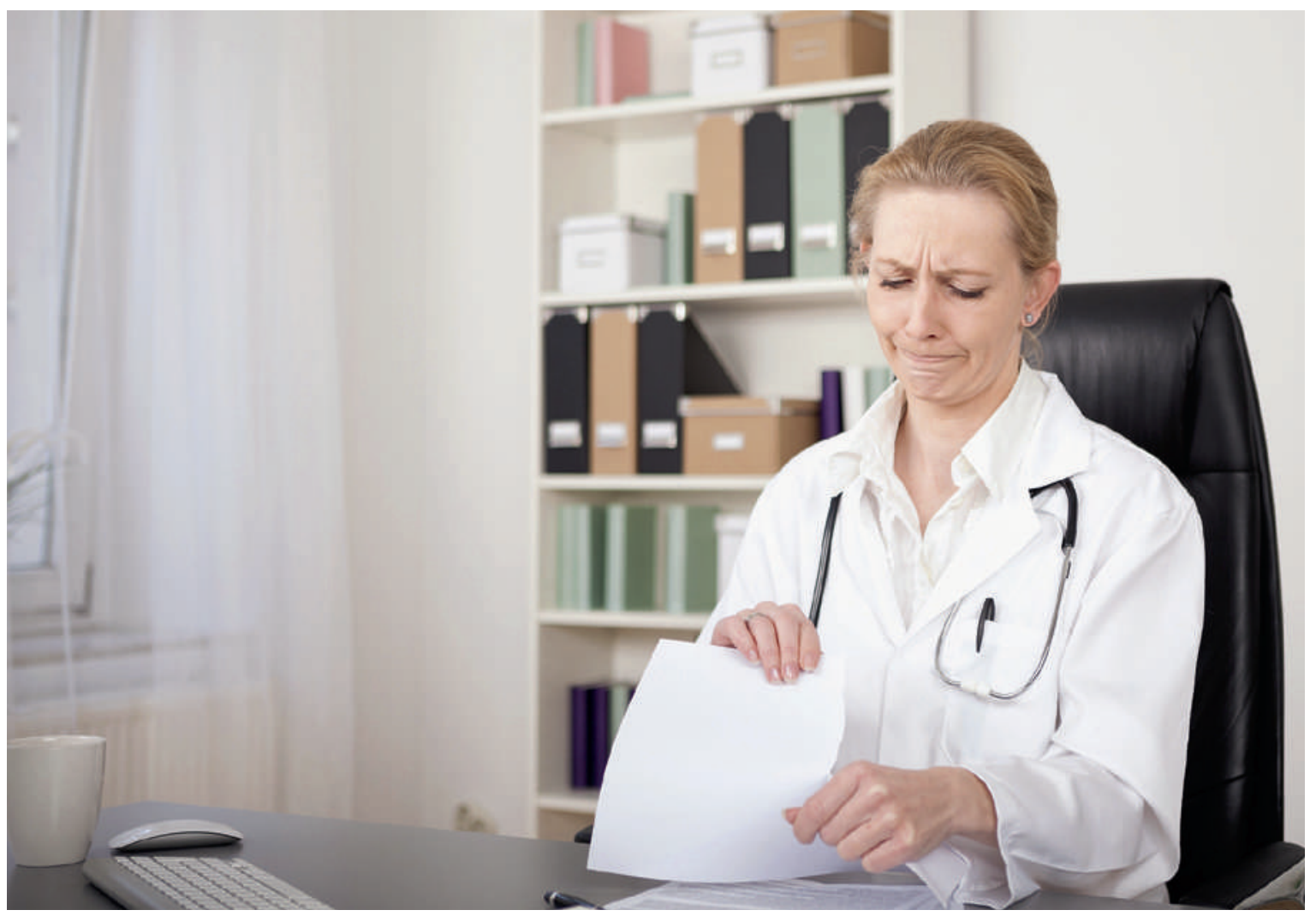

L'échange par écrit avec l'Al peut provoquer de l'irritation chez les médecins. 
groupe, des interviews et des ateliers avec des représentants du corps médical, des offices AI et des services médicaux régionaux (SMR).

\section{Une collaboration variable d'un cas à un autre}

Le mécontentement du corps médical à l'égard de l'AI [2, 3], dont les médias se sont fait l'écho, est également perceptible dans les résultats de l'enquête susmentionnée. Certes, offices AI et médecins interrogés se montrent dans la plupart des cas plutôt, voire entièrement satisfaits de la collaboration. Le degré de satisfaction des deux partenaires est toutefois très variable. En ce qui concerne les mesures médicales pour les assurés de moins de 20 ans, les personnes interrogées se sont déclarées plutôt, voire très satisfaites de la collaboration dans plus de trois cas sur quatre. Ce niveau de satisfaction chute à un peu plus d'un cas sur deux en ce qui concerne l'examen du droit à la rente. Il se situe entre ces deux valeurs lorsque l'AI procède à l'examen des possibilités de réadaptation ou lorsqu'elle vérifie si les circonstances déterminantes pour le calcul d'une rente ont changé. Il ressort de l'enquête que les médecins de famille et les psychiatres sont nettement moins satisfaits de la collaboration que les représentants d'autres spécialités médicales.

\section{Différences dans l'évaluation de la capacité de travail}

L'évaluation de la capacité de travail est une source importante de désaccords entre les partenaires. Pour déterminer dans quelle mesure une personne peut encore travailler, les médecins se servent en effet d'une conception de la santé et de la maladie qui tient davan-

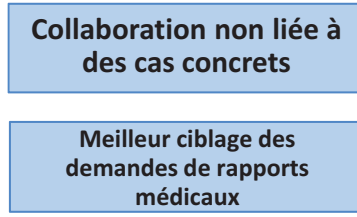

Amélioration des échanges

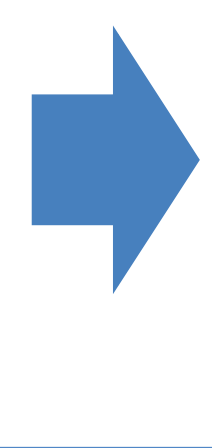

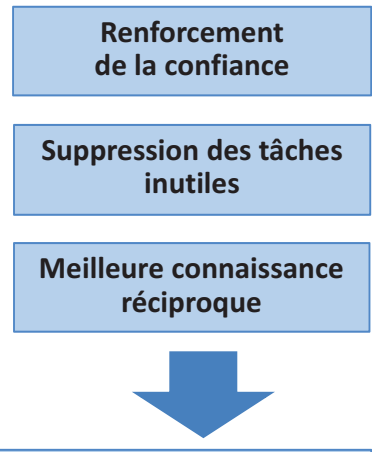

rets
Figure 1: Une collaboration indépendamment du travail sur des cas concrets, un meilleur ciblage des demandes de rapports médicaux et une amélioration des échanges facilitent la collaboration tout en renforçant la confiance mutuelle et la connaissance réciproque des collaborateurs de I'Al et des médecins traitants. tage compte des aspects sociaux que ne le fait celle de l'AI. Ils tendent à évaluer la capacité de travail sur la base d'une approche purement médicale, en considérant la profession actuelle de leur patient et leur propre vision du marché du travail. L'AI examine quant à elle si l'assuré peut encore exercer une activité professionnelle adaptée à son handicap; elle ne compare en fin de compte pas la capacité de travail, mais le salaire réalisable avant et après la survenance du handicap en

L'AI ne compare en fin de compte pas la capacité de travail, mais le salaire réalisable avant et après la survenance du handicap.

considérant non le marché du travail effectif, mais la vision théorique d'un marché du travail équilibré. Enfin, les exigences de l'AI pour la reconnaissance d'une maladie sont plus élevées que celles des médecins traitants, ce qui favorise des appréciations divergentes, surtout dans le cas des maladies psychiques et des affections douloureuses sans cause univoque.

\section{Les médecins ne s'estiment pas pris suffisamment au sérieux}

Les deux partenaires de la collaboration nourrissent par ailleurs des doutes quant à la pertinence de leurs évaluations respectives. Les offices AI reprochent aux rapports médicaux de ne pas toujours être suffisamment précis et mettent parfois en doute l'indépendance des médecins traitants. Ces derniers soulignent quant à eux qu'ils sont, dans la procédure, les seuls médecins dont le jugement s'appuie sur des observations à long terme de l'assuré. Ils émettent également de sérieuses réserves quant à la qualité et l'indépendance des expertises externes.

Sont également mentionnés une méconnaissance de la procédure et des instruments de l'AI par les médecins traitants, un manque de communication directe (une lacune admise par les deux parties), une durée excessive de la procédure et un temps d'attente parfois trop important du rapport médical.

\section{Approches pour renforcer la collaboration}

Leétude a dégagé trois pistes qui, à la lumière de l'expérience passée, sont de nature à améliorer la collaboration avec les offices AI (fig. 1). Ces approches permettent aux partenaires d'améliorer leur travail sur des cas concrets, de renforcer leur confiance mutuelle, de mieux connaître leurs compétences, leurs tâches et leurs responsabilités respectives, et, pour les médecins, de réduire le temps consacré à cette collaboration. 
- Un meilleur ciblage des demandes de rapports médicaux: l'office AI ne devrait pas demander systématiquement des rapports médicaux écrits, mais seulement lorsque cela s'avère nécessaire à la lumière des informations déjà en sa possession (entretiens, rapports existants, par ex. ceux d'autres institutions). Il devrait, dans la mesure du possible, n'en demander qu'aux médecins susceptibles de formuler un avis pertinent sur le cas d'espèce.

Les médecins émettent également de sérieuses réserves quant à la qualité et l'indépendance des expertises externes.

- Une amélioration des échanges: au-delà des demandes de rapports écrits, d'autres échanges entre l'officeAI et les médecins traitants peuvent s'avérer nécessaires pour l'un ou l'autre des partenaires, par exemple dans le but d'obtenir des informations complémentaires, de coordonner la procédure AI avec le traitement médical ou de tirer au clair les divergences d'appréciation. Il serait par l'échange (téléphone, courriel, séance) en fonction de la situation et d'assurer en commun le suivi et la coordination des mesures de réadaptation. En ce qui concerne les éventuelles divergences d'appréciation et la coordination de la procédure AI avec le traitement médical, il faudrait veiller à informer le médecin traitant des principales étapes de la procédure (début d'une mesure, expertise, préavis, décision). Il importe à cet égard de clarifier le rôle qui revient à l'assuré dans cet échange d'informations et de renforcer la protection de ses droits de la personnalité.

Correspondance:

Christian Bollige

Bureau Vatter SA

Gerberngasse 27

CH-3011 Berne

Tél. 0313126575

bolliger[at]buerovatter.ch sur des cas concrets: les offices AI devraient, lors de l'organisation et de la réalisation de manifestations de formation et d'information, avoir recours aux caailleurs souhaitable de définir les modalités de

naux de communication existants de la profession médicale, par exemple aux cercles de qualité et de formation continue à l'échelle régionale. L'étude a également identifié des formes plus poussées de collaboration non liées à des cas concrets. C'est ainsi qu'un office AI a élaboré une convention de collaboration avec une société de discipline médicale.

\section{Conclusion}

Les différentes pistes qui viennent d'être évoquées visent à réduire à l'essentiel la communication écrite et à encourager les contacts personnels directs lorsque ceux-ci permettent un gain de temps. Les différences fondamentales existant entre la pratique médicale et la médecine des assurances continueront d'entraîner des divergences dans les appréciations portées sur la

\section{Il faut réduire à l'essentiel la communication écrite et encourager les contacts personnels directs.}

capacité de gain des assurés dans le cadre de la procédure AI. Une estime réciproque entre les partenaires peut néanmoins aider, dans de telles situations, à assurer un échange efficace des informations, un déroulement adéquat et efficace de la procédureAI, ainsi qu'une meilleure coordination entre celle-ci et le traitement médical.

\section{Références}

1 Bolliger C, Féraud M. Zusammenarbeit zwischen IV-Stelle und behandelndem Arzt: Formen, Instrumente und Einschätzungen der Akteure (Aspects de la sécurité sociale, rapport de recherche $\mathrm{n}^{\circ}$ 5/15). Berne: Office fédéral des assurances sociales; 2015.

2 Romann C. Collaboration avec l'AI: nous sortons enfin de l'impasse. Bull Méd Suisses. 2012;93(11):397.

3 Schilling G. L'avis des médecins de famille n'est pas pertinent pour l'AI. Alors pourquoi nous consulte-t-on au juste? PrimaryCare. 2014;14(21):333.

\section{Crédit photo}

Sebastiangauert | Dreamstime.com 